\title{
Investigation on feasibility of recurrence quantification analysis for detecting flank wear in face milling
}

\author{
S.D. Mhalsekar ${ }^{1 *}$, S.S. Rao ${ }^{2}$, K.V. Gangadharan ${ }^{2}$ \\ ${ }^{1 *}$ Department of Mechanical Engineering, Institute of Shipbuilding Technology, Vasco, Goa, INDIA \\ ${ }^{2}$ Department of Mechanical Engineering, National Institute of Technology Karnataka, Surathkal, INDIA \\ *Corresponding Author: e-mail: shridirgame@gmail.com, Tel +91-512-2597009, Fax.+91-512-2590063
}

\begin{abstract}
Flank wear is a critical phenomenon which has direct impact on quality of surface finish, dimensional precision and ultimately cost of the finished product. In any metal cutting operation, cutting tool wear estimation will help in identifying tool state, which is a critical factor in productivity. In this paper, the vibration signals are used for detecting flank wear in face milling .The vibration signals are analyzed using a novel non linear technique called recurrence quantification analysis (RQA). RQA includes time delay and dimensions embedding process so as to reconstruct the time series data of vibration signal, to obtain better information of the changes in nonlinear dynamics underlying the milling process. An investigation of this technique was carried out to see its capability in detecting flank wear in face milling. Experiments were conducted on universal milling machine using AISI H11 steel as work material. The investigation proved that RQA technique has a good potential in detecting flank wear in face milling. The RQA parameters such as percent recurrence (REC), trapping time (TT), percent laminarity (LAM) and entropy (ENT), and also the recurrence plots color patterns for different flank wear, can be used in detecting insert wear in face milling.
\end{abstract}

Keywords: milling, flank wear, recurrence plot, recurrence quantification analysis.

\section{Introduction}

The productivity of a machining system and machining cost, as well as quality, the integrity of the machined surface and profit strongly depends on tool wear and tool life. Sudden failure of cutting tools leads to loss of productivity, rejection of parts and consequential economic losses. Flank wear occurs on the relief face of the tool and is mainly attributed to the rubbing action of the tool on the machined surface. Since the flank wear predominantly occurs in cutting tools, so the life of a particular tool used in the machining process depends upon the amount of flank wear. The flank face of the cutting tool performs a rubbing action against the work piece materials, the surface finish of the machined work piece gets affected and depends upon the amount of flank wear. An increase in the amount of flank wear leads to a reduction in nose radius of the cutting tool, which in turn reduces the surface finish. The maximum utilization of cutting tool is one of the ways for an industry to reduce its manufacturing cost. Hence, tool wear has to be controlled and should be kept within the desired limits for any machining process. Tool wear mainly depends upon the machining parameters used for milling of a particular work material.

Cutting tool failures (wear and breakage) typically represent about $20 \%$ of the downtime of a machine tool (Karuda and Bradley, 1997). Tool wear is found to have a direct impact on the quality of surface finish, dimensional precision and ultimately cost of the finished product (Sick, 2002). It is found that over-estimation of tool life results in degraded product quality and damaged part (in case of early breakage of the tool), while underestimation leads to frequent stoppage of the machining process and increased cost of production. So, accurate wear estimation in machining processes is a key research topic in automated manufacturing.

There are direct methods of wear estimation such as: visual inspection, measurements of volumetric textures, which are not cost effective and reliable. Researchers are active in finding an efficient and effective tool condition method, and are mainly focusing on indirect methods of estimating wear, which are online and use processed signals, such as cutting forces, current and power for various drives, vibration and acoustic emission (AE), that are known to be significantly affected by the wear phenomenon (Dimla, 
2000). Vibration based monitoring systems are cost effective and represent an effort to prevent catastrophic failure, reduce the costly down time involving unnecessary tool replacement and also to prevent under estimation or overestimation of tool wear. The general philosophy is to look for easy indication (vibrational signature) of a problem such that appropriate action may be taken before more serious problems arise.

The aim of the present research is to examine the appearance of vibration signal and suggesting the method of flank wear detection in real time milling process. Present work investigates, the time series of vibration signal obtained during face milling process while machining with sharp, $0.18 \mathrm{~mm}$ and $0.25 \mathrm{~mm}$ worn milling insert. A nonlinear embedding methods and the recurrence plots (RPs) technique is then applied to the corresponding time series. This technique reveals the changes in nonlinear dynamics underlying the milling process. RQA parameters obtained are investigated for detecting flank wear in face milling. Recurrence analysis is comparatively a new non linear technique developed by (Eckmann et al., 1987). The technique has been successfully applied to different fields ranging from physiology (Webber and Zbilut, 1994) to economics (Belaire-Franch, 2004). Recently it has made forays into engineering field. One of the first, had application of RPs to time series generated by models of the Twin-T, Wien-bridge and other chaos generating electronic oscillator circuits (Elwakil and Soliman,1999). Through visual inspection of the RPs, the chaotic behavior of the model results was confirmed. RPs has been used to estimate optimal embedding parameters and vicinity threshold. It was used as a noise reduction scheme in human speech signals (Matassini and Manfredi, 2002). (Litak et al., 2008) investigated the time series of a torque applied to the ripping head in the process of a cutting concrete rock with sharp and blunt tools. By applying nonlinear embedding methods and the recurrence plots technique to the corresponding time series, the changes in nonlinear dynamics lying behind the ripping process were indicated. Litak has proposed of using this technique for testing of the ripping machine efficiency and a method to monitor the state of tools. (Nichols et al, 2006) have mentioned that RQA as a useful tool for detecting subtle non stationarities and/or changes in time series. It has been applied to detect damage induced changes to the structural dynamics. Two of the RQA parameters were found to be sensitive to damage than are the plate frequencies. (Masao, 2006) in his paper has presented recurrence plot scheme approach to analyze nonstationary transition patterns of IP-network traffic. In performing a quantitative assessment of dynamical transition patterns of IP-network traffic, the values of determinism (DET) defined by the recurrence quantification analysis (RQA) was used. (Sen $e t$ al., 2008) studied the fluctuations of mean indicated pressure (MIP) in a diesel engine using recurrence plots, recurrence quantification analysis and continuous wavelet transform. Sen has proposed the use of results of their study in developing effective control strategies for efficient engine performance. (Gonzáleza et al., 2008) analysed the electrochemical current oscillations generated during the early stages of corrosion fatigue damage using RPs and RQA and could separate the electrochemical process of pitting corrosion from the corrosion fatigue crack initiation and growth. Since recurrence analysis technique has been sparsely used in engineering application and also it is suitable in detecting subtle changes in dynamic behavior. This fairly new concept in engineering field can be exploited in investigating cutting operation dynamics.

\section{Recurrence Plot}

The RP approach provides a qualitative interpretation of hidden patterns of dynamical systems (Eckmann et al., 1987). It enables one to investigate the dimensional phase space trajectory by means of two-dimensional (2-D) visualization. By using RPs, we can efficiently check variation patterns of time-series data. Recurrence Plots are graphical tools based on Phase Space Reconstruction. The changing state of a dynamic system can be represented by sequences of 'state vectors' in the phase space.

Consider an acceleration time series data from a vibration signal:

$$
a=\{a(1), a(2), a(3) \ldots . ., a(T)\}
$$

Where $\mathrm{T}$ is the length of the time series data.

From this single one dimensional data vector a family of new vectors called as embedded vectors is to be constructed each having length $m<T$ which is as given below:

$$
A_{i}=\{a(i), a(i+\tau), \ldots . ., a(i+(m-1) \tau)\}
$$

Where $i=1$ to $T, \tau$ is the delay time and $m$ is the embedding dimension.

The number of new vectors is given by:

$$
N=T-(m-1) \tau
$$

Then, the distance between two state vectors $A_{i}$ and $A_{j}$ can be defined as follows:

$$
D_{i j}=\left|A_{i}-A_{j}\right|
$$


The RP visualizes the relationships between two state vectors within a 2-D square matrix of size $T \times T$. In this process, a threshold distance $\varepsilon$ is generally set for plotting recurrence points, which fall in the neighborhood of a fixed size.

Mathematically it is defined as:

$$
r_{i j}=\theta\left(\varepsilon-\left\|A_{i}-A_{j}\right\|\right)
$$

Where $r_{i j}$ an element of the recurrence matrix is, $\varepsilon$ is the threshold for the distances, \|\|$\|$ is a norm, and $\theta$ is the heaviside function, defined as:

$$
\begin{aligned}
& \theta(x)=0, \text { for } x<0 \\
& \theta(x)=1, \text { for } x \geq 0
\end{aligned}
$$

In other words, $r_{i j}$ assumes a value of 1 if $(i, j)$ is recurrent, and a value of 0 otherwise, which are plotted as black and white. Instead of plotting the recurrences in two values (i.e., black and white) by setting a threshold level, the $D_{i j}$ distance matrix can also be used, which helps checking the phase space trajectory of target data (Dissinger et al. 1992). These distances are given color codes and the RPs occurs as color patterns. With proper values of $m$ and $\tau$ this representation is topologically equivalent to the underlying $m$ dimensional system that produced the time series (Zbilut and Webber, 1992). Therefore each unknown point of the phase space at time $i$ is reconstructed by the delayed vector in an $n$-dimensional space called the reconstructed phase space. There exist a variety of methods for choosing values of $m$ and $\tau$. The optimal delay will produce independent (in the statistical sense) pseudo-state vectors while the optimal dimension will be the smallest one that prevents trajectories in state space from crossing. The most often used methods are the Average Mutual Information (AMI) function for computing the time delay $\tau$, as introduced by (Fraser and Swinney, 1986) and the False Nearest Neighbors (FNN) method for the embedding dimension $m$ developed by (Kennel et al, 1992). One chooses the first minimum location of the average mutual information function as $\tau$. The percentage false nearest neighbors (FNN) is computed for each of the $m$ values. The embedding dimension is said to be found for the first percentage of FNN dropping to zero. This percentage never reaches a true zero value because of presence of noise in the signal. Hence optimal $m$ is chosen for minimum value of FNN. The embedding parameters can be found out using the Visual Recurrence Analysis (VRA) version 4.6 by Eugene Kononov, which is available on the internet.

\section{Recurrence Quantification Analysis}

The RP was able to provide only qualitative visual information. Later in order to quantify the lines in RP a way of measuring quantitative information has been introduced (Zbilut and Webber, 1992). This provided the means for recurrence quantification analysis. It defines measures of complexity. Following are quantitative parameters of RQA defined as:

Percent recurrence REC, measures the percentage of recurrent points in the phase space, excluding the main diagonal. Embedded processes that are periodic have higher percent recurrence values.

$$
R E C=\frac{1}{N^{2}} \sum_{i, j=1}^{N} r_{i j}
$$

Percent determinism DET is the percentage of recurrent points that are included in line segments parallel to the upward diagonal and whose length meets or exceeds the minimum length threshold. It allows distinguishing between dispersed recurrent points and those that are organized in diagonal patterns, representing strings of vectors (deterministically) repeating themselves.

$$
D E T=\frac{\sum_{l=l_{\min }}^{N} l . P(l)}{\sum_{i, j=1}^{N} r_{i j}}
$$

where $l$ is the length of the diagonal line parallel to the Line of Identity, $P(l)$ is the frequency distribution of the diagonal lines (that is the histogram of the their lengths $l$ ) and $r_{i j}$, the recurrence of a state.

Percent laminarity LAM is the percentage of recurrent points that are included in line segments vertical to the upward diagonal and whose length meets or exceeds the minimum length threshold. It measure chaotic transitions, is related with the amount of laminar phases in the system (intermittency). 


$$
L A M=\frac{\sum_{v=v_{\min }}^{N} v \cdot P(v)}{\sum_{v=1}^{N} v \cdot P(v)}
$$

Where $P(v)$ is the frequency distribution of the lengths $v$ of the vertical lines, which have at least a length of $v_{\min }$.

Trapping time TT is the average length of vertical line structures. It represents the average time in which the system is "trapped" in a specific state.

$$
\mathrm{TT}=\text { average length of vertical lines }
$$

Ratio variable is defined as the quotient of DET divided by REC. It is useful to detect transitions between states. This ratio increases during transitions but settles down when a new quasi-steady state is achieved.

Entropy ENT variable, is computed as the Shannon entropy of the frequency distribution of the diagonal line lengths distributed over integer bins in a histogram, and quantifies the complexity of recurrence plots. More precisely, the entropy gives a measure of how much information one needs in order to recover the system. A low entropy value indicates that little information is needed to identify the system; in contrast, high entropy indicates that much information is required. Upward line segment lengths are counted and distributed over integer bins of a histogram. Shannon entropy is computed according to the formula:

$$
E N T=-\sum_{l=I_{\operatorname{lin}}}^{N} P_{l} \log _{2}\left(P_{l}\right)
$$

Maxline The longest line segment measured parallel to the main diagonal. A periodic signal produces long line segments, while short lines indicate chaos.

Trend variable is the slope of the least squares regression of local recurrence as a function of the orthogonal displacement from the main diagonal. It represents the measure of the positioning of recurrent points away from the central diagonal. It quantifies the degree of system stationarity. In other words it represents the measure of the positioning of recurrent points away from the central diagonal. Low value of trend indicates stationarity in the signal and high value of trend, positive or negative indicates, drift in the signal.

\section{Experiments}

Experiment was conducted on universal milling machine with single carbide insert fixed to the cutter. Single insert cutter was used in the experiment to avoid run out effect of insert during milling. Vibration signals were acquired using National Instruments NI9234 DAQ system (2008) with LabVIEW 8.6 Software. Vibration signals were acquired using MMF KS943L IEPE triaxial accelerometer of Metra Mess- und Frequenztechnik (2007) which had $14.23 \mathrm{mV} / \mathrm{g}$ voltage sensitivity and range up to $240 \mathrm{~g}$. Following cutting conditions were maintained:

Table 1. Cutting Conditions

\begin{tabular}{|c|c|c|}
\hline Conditions & I & II \\
\hline Speed (rpm) & 300 & 510 \\
\hline Depth of cut (mm) & 0.5 & 0.5 \\
\hline Feed (mm/min) & 22.5 & 22.5 \\
\hline
\end{tabular}

In order to investigate tool wear detection using RQA, experimental results were used. The experiment has been carried out on a conventional universal face milling machine with $5 \mathrm{hp}$ spindle motor and $3 \mathrm{hp}$ feed motor. Two accelerometers were used to measure vibration signals. One was mounted on the fixture holding work material and the other on spindle housing. The measurements were sampled at 2000 points/second. A simple block diagram in LabVIEW was constructed to acquire the vibration data and is shown in the Figure 1. 


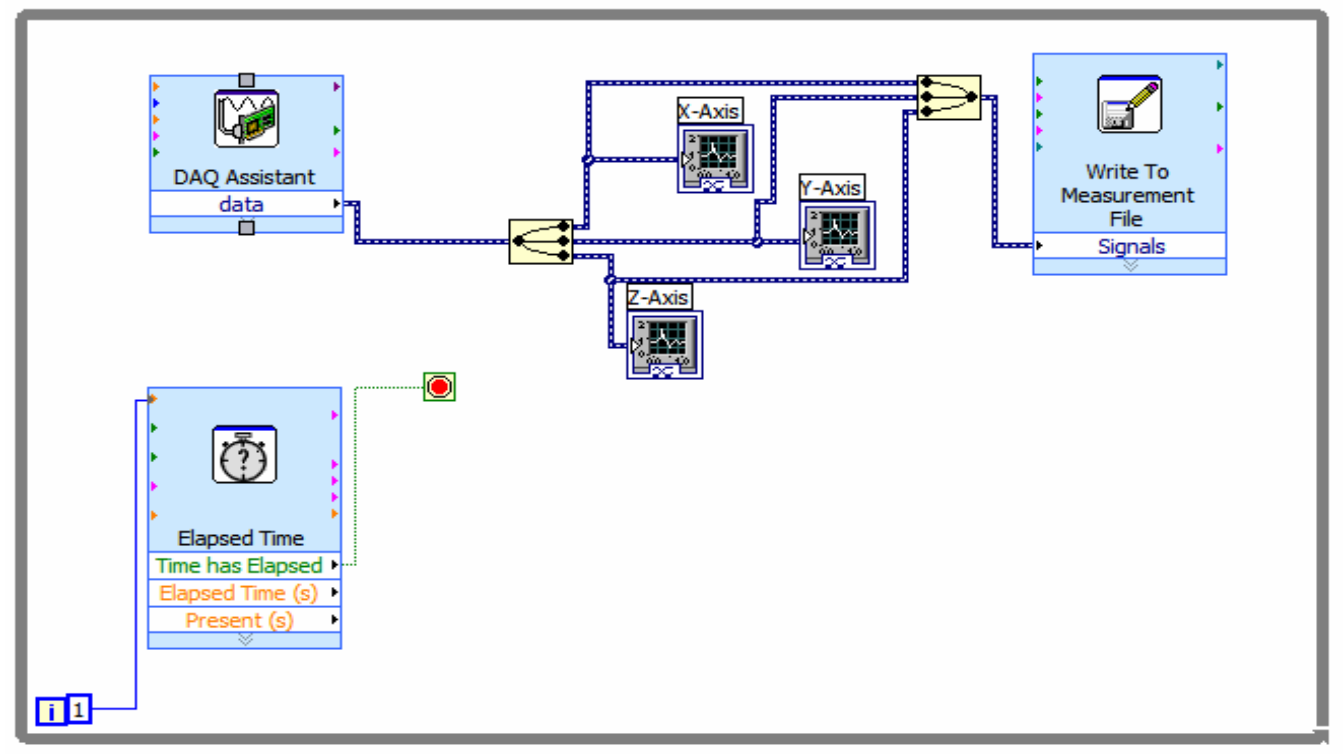

Figure 1: Block diagram of LabVIEW program for Data Acquisition

The first 500 points were selected for analysis. It was selected in a way that at least two revolutions of the cutter were included. The digital data from the A/D board were then acquired and stored by the LabVIEW software into two files for the two vibration components of each transducer. Three states of tool wear were considered i.e. sharp, $0.18 \mathrm{~mm}$ wear and $0.25 \mathrm{~mm}$ wear. Work piece material used in the experimental trials is $\mathrm{H} 11$ hot rolled chromium steel. All the specimens were of rectangular cross section and having 60 X 100 X 22 mm dimensions. The milling cutter and inserts used were of SECO R220.43-0063-07W and 07045 TN M16 $373 \mathrm{M}$ respectively. Figure 2 shows the experimental setup.

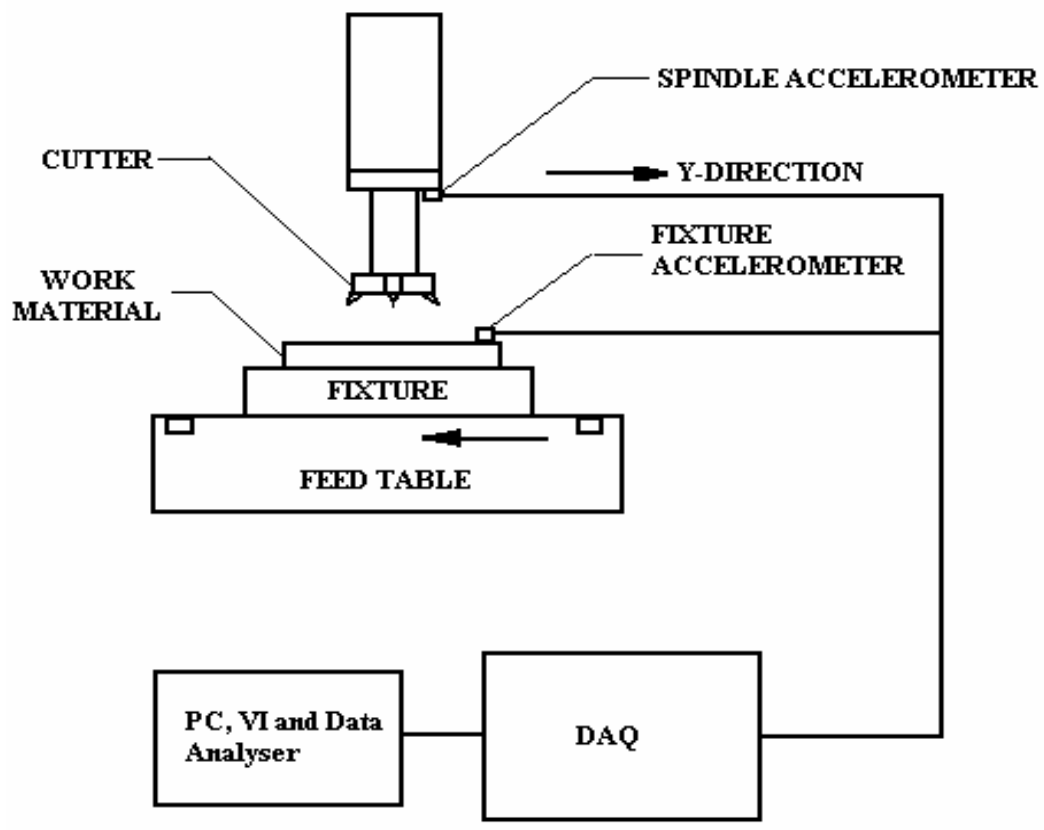

Figure 2: Experimental set up 
Data stored in LabVIEW files were analyzed using VRA software. Only Y- direction data obtained from accelerometer mounted on the spindle was used in the investigative study. This direction is in feed direction and is most sensitive compared to the data obtained in other directions.

\section{Results and Discussion}

A detailed acceleration-time series plot were obtained for milling with a sharp insert, 0.18 mm worn insert and $0.25 \mathrm{~mm}$ worn insert, as shown in Figure 3 for the two cutting conditions respectively. The $\mathrm{Y}$ axis representing acceleration in $\mathrm{g}$ m/sec ${ }^{2}$ and $\mathrm{X}$ axis indicates number of samples. These plots were obtained using VRA software.
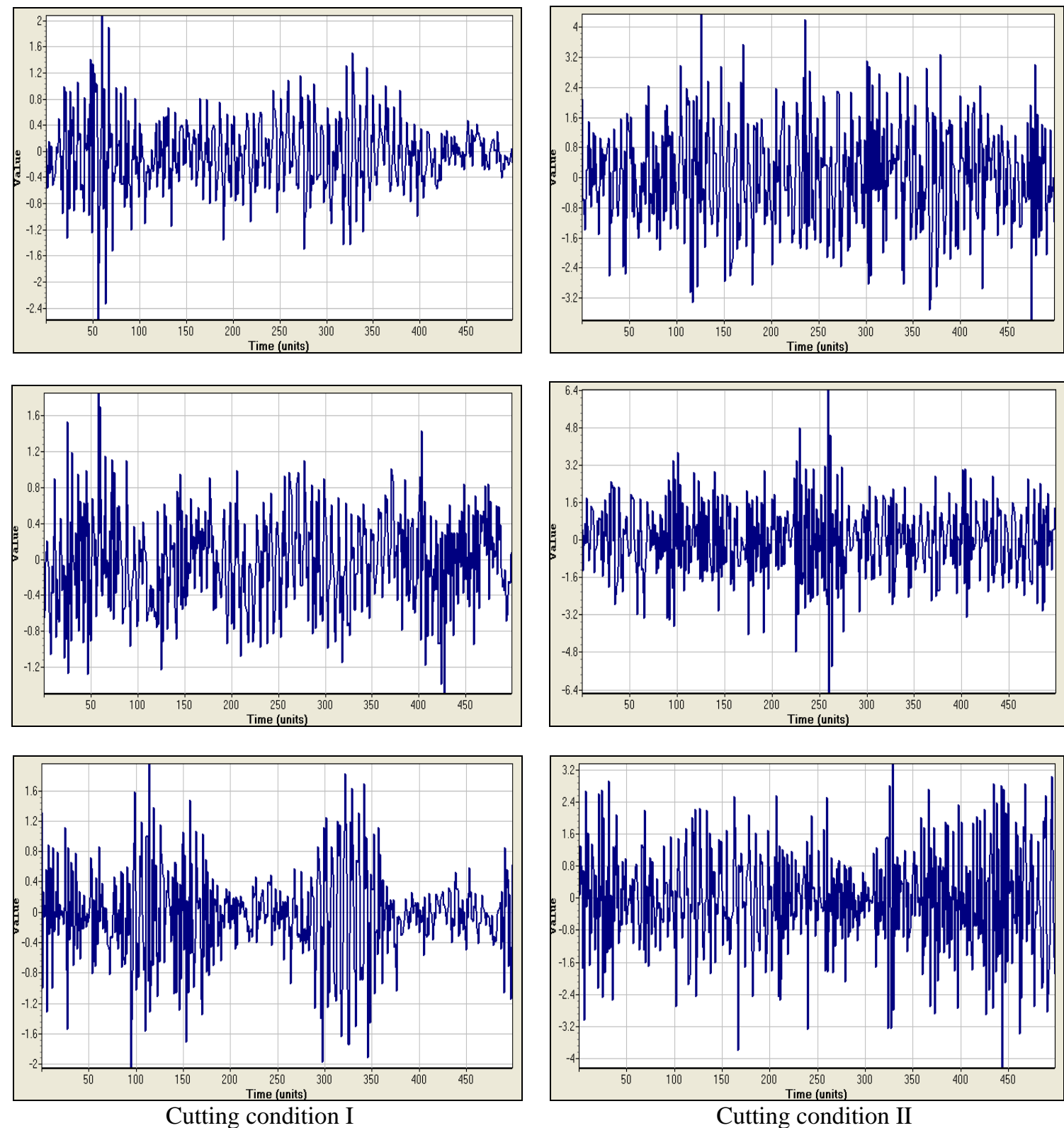

Figure 3: Time series plot of vibration data for sharp insert, $0.18 \mathrm{~mm}$ worn insert and $0.25 \mathrm{~mm}$ worn insert during cutting condition I and condition II

The times series plots of vibration acceleration for sharp tools are characterized by slightly larger peak values while the worn inserts shows shorter amplitude of acceleration. This does not provide clear and distinct method to identify the wear states of the cutting inserts. The acquired time series data are further analyzed using VRA software to generate unthreshold RPs. 
The procedure followed to get RP and RQA values was:

- Average Mutual Information analysis was performed to get embedded time delay value.

- False Nearest Neighbor analysis is carried out to determine embedded dimension using previously obtained time delay value. The maximum value for embedded dimension was fixed at 15.

- Recurrence plot without threshold was generated using the available values of embedded time delay and dimension.

- RQA analysis was carried out using time delay, dimension and threshold radius. The threshold radius of 1.5 was maintained constant throughout the analysis.

As per this procedure using the VRA software AMI and FNN estimated the embedding parameters for the time series of sharp insert, $0.18 \mathrm{~mm}$ worn insert and $0.25 \mathrm{~mm}$ worn insert. In Figure 4 one can easily find that the optimal delay as $\tau=2$ from AMI graph. The time delay is selected at first minimum value of AMI plot.

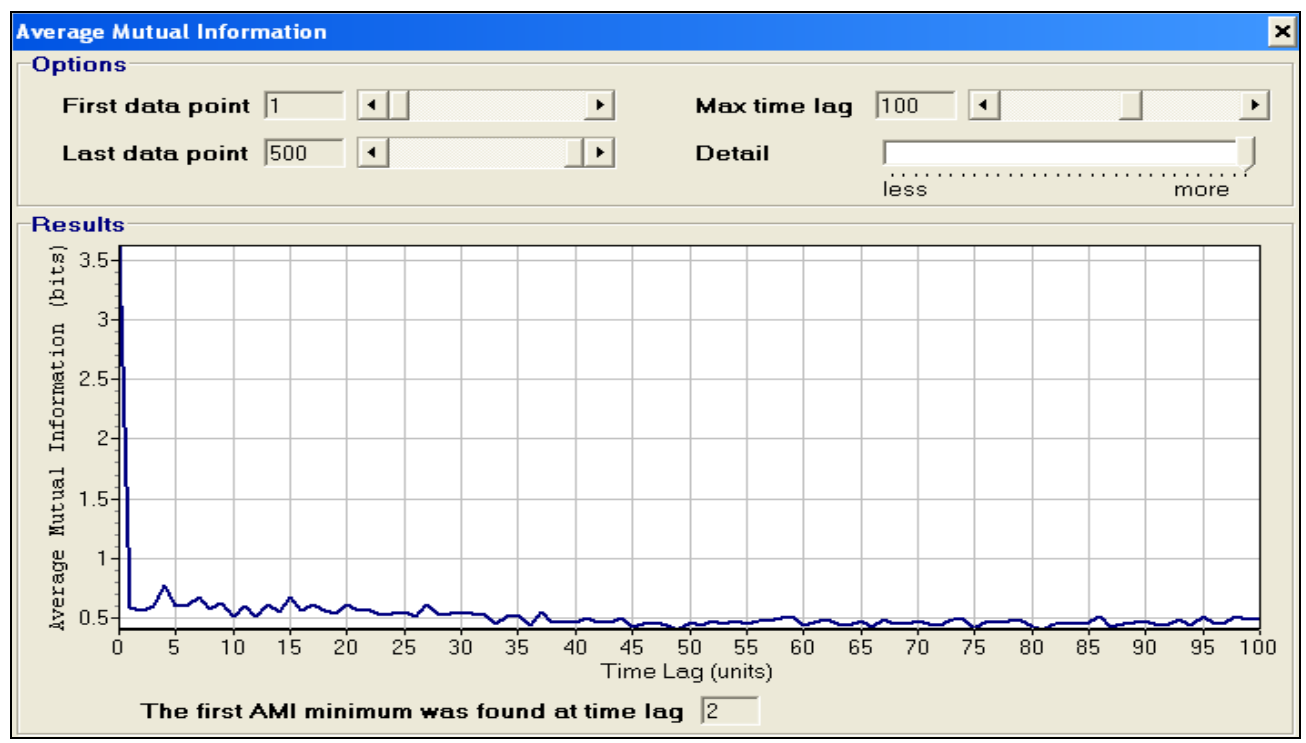

Figure 4: AMI plot for condition I using sharp insert

This time delay is used in getting the embedding dimension. Figure 5 shows the embedding dimension $m=15$ obtained from FNN Graph when time delay is 2 for cutting condition I with sharp insert. The embedding dimension is obtained as first minimum value of FNN plot.

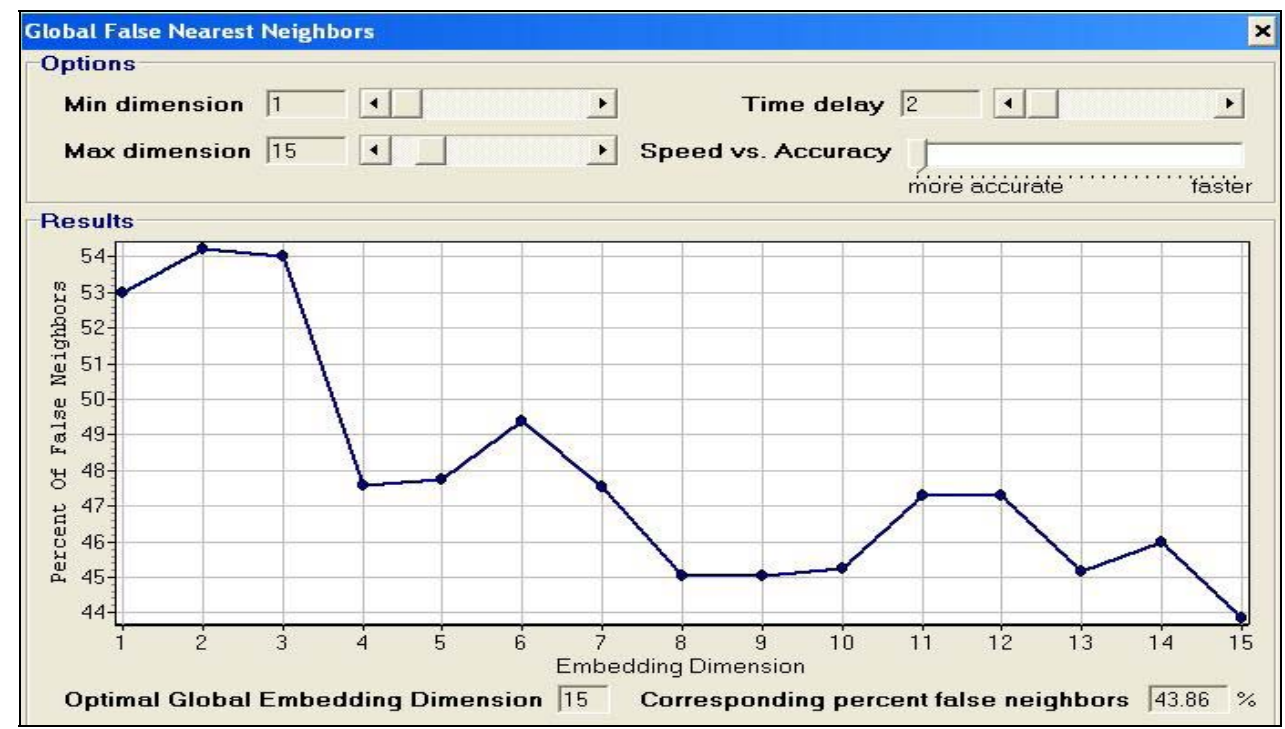

Figure 5: FNN plot for condition I using sharp insert 
In this manner AMI and FNN analysis for both the cutting conditions with the three types of wear is carried out. The corresponding values of embedding dimension and time delay are tabulated as shown in Table 2. These values provide base for constructing the phase space to reveal the underlying milling dynamics when milling with different flank worn inserts. Table 2 indicates the values of embedding dimension and time delay are not sufficient to identify the wear levels for the two cutting conditions. The embedding dimension for cutting condition II and $0.18 \mathrm{~mm}$ wear reduced drastically to 9 as seen in Table 2 . It could be due to presence of hard particle or void in the work material, which gave rise to a small change in milling dynamics.

Table 2: Embedding dimension, time delay and threshold radius

\begin{tabular}{|c|c|c|c|}
\hline \multicolumn{5}{|c|}{ For Cutting condition I } \\
\hline & $\mathrm{m}$ & $\tau$ & $\varepsilon$ \\
\hline Sharp & 15 & 2 & 1.5 \\
\hline $0.18 \mathrm{~mm}$ wear & 15 & 2 & 1.5 \\
\hline $0.25 \mathrm{~mm}$ wear & 11 & 2 & 1.5 \\
\hline \multicolumn{5}{|c|}{ For Cutting condition II } & $\varepsilon$ \\
\hline Sharp & $\mathrm{m}$ & $\tau$ & 1.5 \\
\hline $0.18 \mathrm{~mm}$ wear & 15 & 2 & 1.5 \\
\hline $0.25 \mathrm{~mm}$ wear & 9 & 2 & 1.5 \\
\hline
\end{tabular}

These results would be a framework for the construction of RP plots and RQA techniques.

\subsection{Qualitative Investigation}

Using the results of AMI and FNN, the corresponding recurrence plots were developed without considering threshold radius as shown in Figure 6 and Figure 7 for the two cutting conditions and the three wear states of the inserts A clear distinction in the patterns of RP is seen. Information about the dynamics of a time series is usually obtained from the line structure and point density in a RP. A homogeneous RP with no structure is typical of a stationary or autonomous process such as white noise. RPs of oscillating systems, on the other hand, has diagonally oriented or periodic recurrent structures (i.e., diagonal lines or checkerboard patterns). Vertical or horizontal lines in a RP signify the presence of laminarity or intermittency in the time series, whereas abrupt changes in dynamics as well as extreme events are characterized by white areas or bands (Marwan, 2003).

These textures of RPs are required to provide initial visual inspection and in identifying the changes in dynamics of the system. As inserts with different wear level are performing cutting operation , the vibration signals will be having different dynamics which are revealed by color or/and texture pattern of RPs. Figure 6 and Figure 7 reveal the changing RP patterns for different cutting conditions and wear levels.

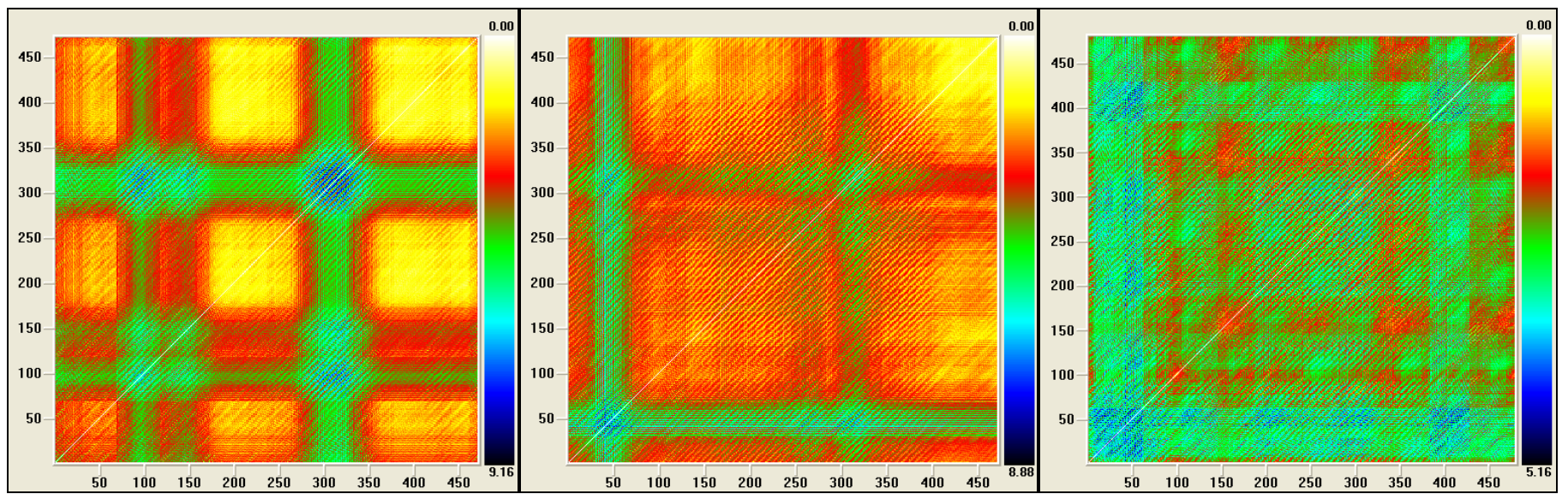

Figure 6: Recurrence plots for sharp, $0.18 \mathrm{~mm}$ worn and $0.25 \mathrm{~mm}$ worn inserts during cutting condition I 


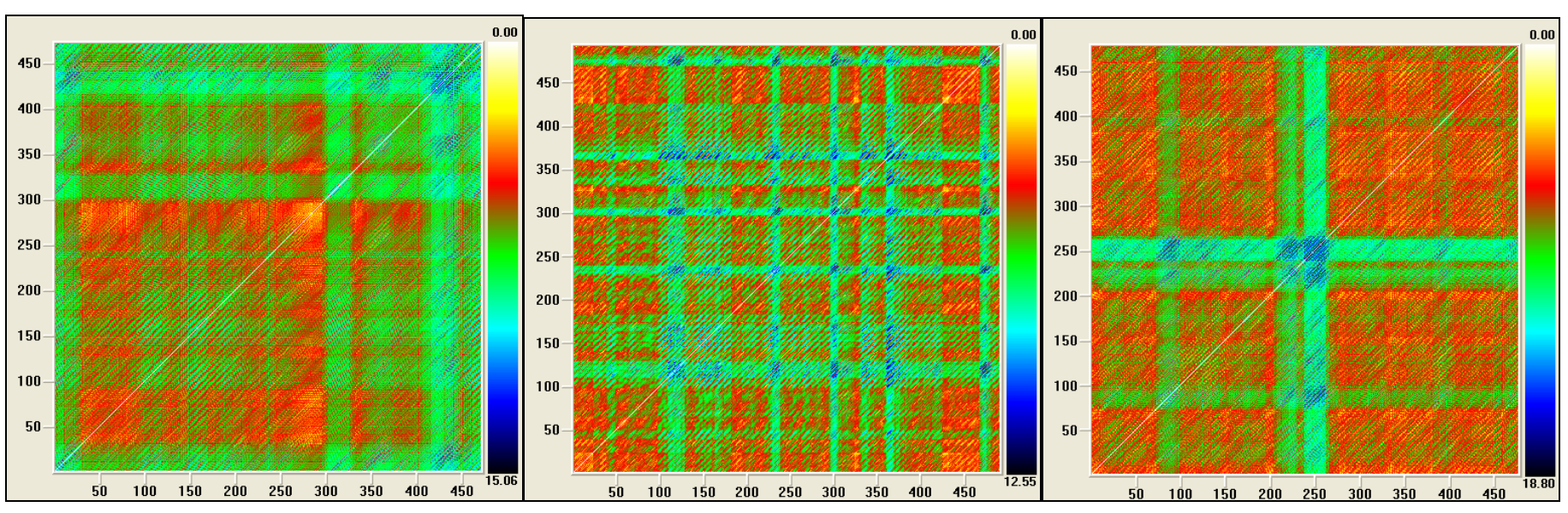

Figure 7: Recurrence plots for sharp, $0.18 \mathrm{~mm}$ worn and $0.25 \mathrm{~mm}$ worn inserts during cutting condition II

RPs in Figure 6 shows lot of diagonal lines thus indicating periodicity in milling operation Presence of vertical lines is more for $0.25 \mathrm{~mm}$ wear indicating laminarity or intermittency in the milling process as wear has increased to a greater extent. For this wear it is seen from RP that the shorter diagonal lines which are away from the main diagonal line called as line of identity are showing lot of the discontinuities in them as compared to sharp and $0.18 \mathrm{~mm}$ worn insert RPs. There is clear change in colour combinations of the RP patterns as RPs changes from sharp to $0.25 \mathrm{~mm}$ wear inserts. This was qualitative observation for cutting condition I.

Similar type of features can be observed in RP patterns of cutting condition II as shown in Figure 7.In RP patterns for condition II, lot of vertical lines as compared to RP patterns of condition I are present, indicating more presence of intermittency as compared to cutting condition II, since in condition II the speed is at a higher level $(510 \mathrm{rpm})$. The presence of diagonal lines parallel to each other indicates periodicity since cutting in milling operation is regular and periodic. In the second condition also the discontinuities in diagonal lines increases in the RP patterns from sharp to $0.25 \mathrm{~mm}$ wear. As wear progresses the instability in milling dynamics increases which shows the effect on RP plots. The colour combination of RPs also changes. The features described for RP indicate the characteristics in identifying the different wear levels of milling inserts. In this investigation study, the RPs was able to provide a visual comparison between different wear levels. This completes the qualitative investigation of RPs in detecting flank wear.

\subsection{Quantitative Investigation}

RPs provides the initial visual comparison between different state of the insert wear condition. The quantitative investigation of RPs is required to substantiate the qualitative observations. The RPs has to be quantified for which RQA provides the frame work. RQA parameter investigative study was carried out for cutting condition I and II and for different wear inserts. Figure 8 shows RQA analyses for a sharp insert with cutting condition I using VRA software. The embedding dimension, time delay and threshold radius as indicated in Table 2 were used in RQA analysis. Euclidian distance was considered for determining distance between new vectors. A minimum diagonal length of 2 units was considered as base for RQA analysis.

Figure 8 shows the evolution section, distances section and phase space section. The dimension, delay is fixed based on AMI and FNN analysis. A Euclidean distance is chosen for computing the distances between the phase space vectors. The RQA analysis is carried out for full data after phase space reconstruction i. e. from 1 to 488 which is called as full epoch study. RQA parameters are computed for the full epoch and recorded. The full epoch study is carried on vibration data of both cutting conditions with different wear levels of the milling inserts. This will help in bringing out the influence of insert wear on RQA parameter and also the behavior of RQA parameters as cutting condition changes. 


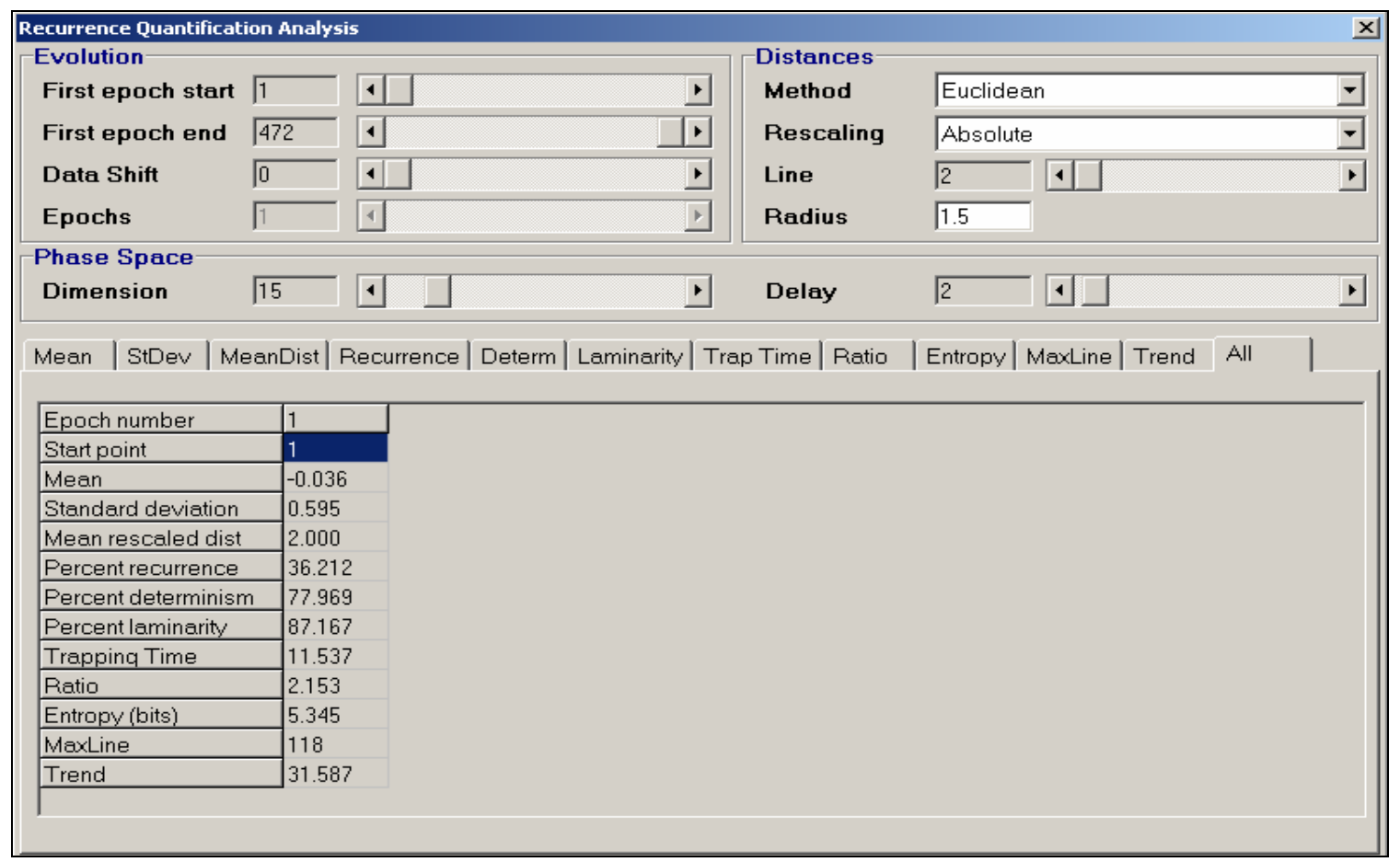

Figure 8: Recurrence Quantification Analysis for sharp insert during cutting condition

The summary of RQA parameters is shown in Table 3 and Table 4. The variation of RQA parameters as wear increases is shown with the help of RQA parameter graphs for cutting conditions I and II respectively as shown in Figure 9 and Figure 10 respectively.

Table 3: RQA analysis for cutting condition I

\begin{tabular}{|c|c|c|c|}
\hline \multicolumn{5}{|c|}{ Cutting condition I } \\
\hline Tool state & sharp & $0.18 \mathrm{~mm}$ & $0.25 \mathrm{~mm}$ \\
\hline Percent recurrence & 36.212 & 27.607 & 18.319 \\
\hline Percent determinism & 77.969 & 58.458 & 43.082 \\
\hline Percent laminarity & 87.167 & 68.215 & 53.055 \\
\hline Trapping time & 11.537 & 5.394 & 3.851 \\
\hline Ratio & 2.153 & 2.117 & 2.352 \\
\hline Entropy & 5.345 & 4.137 & 3.634 \\
\hline Maxline & 118 & 51 & 53 \\
\hline Trend & 31.587 & -12.876 & 5.87 \\
\hline
\end{tabular}

\subsubsection{Influence of wear on RQA parameter during cutting condition I}

Table 3 and Figure 9 shows the influence of wear on RQA parameters during cutting condition I

- REC : It is observed that as insert flank wear level increases from sharp, $0.18 \mathrm{~mm}$ and $0.25 \mathrm{~mm}$, the REC values decreases as found from the REC plot shown in Figure 9. Embedded processes that are periodic have higher percent 
recurrence values. Milling with sharp insert is more periodic compared to worn insert milling; this correlation is well depicted by REC values.

- DET: DET values show a decreasing trend as wear level increases. DET values indicate deterministic nature of the embedded series. For sharp insert DET value is highest and hence the embedded time series is quite deterministic compared to that of $0.18 \mathrm{~mm}$ and $0.25 \mathrm{~mm}$ worn inserts. This is due to increased flank wear on the inserts, which results in machining dynamics becoming more complex and hence the embedded time series becomes less deterministic.

- LAM: The LAM values indicate intermittency or laminarity in the process. In dynamical systems intermittency is the alternation of phases of apparently periodic and chaotic dynamics. The LAM values are decreasing as wear values increase in the cutting condition I. LAM values for sharp is high indicating extent of intermittency is more when milling with sharp inserts than with worn insert. The periodicity during milling with sharp insert is more as depicted from REC values. So, it means it is most of the time in periodic phase than in chaotic phase.

- TT: The TT values show decreasing trend as wear level is increasing. As TT values indicate the average time the system is trapped in specific state, it can be deduced for sharp insert that the system is trapped for more amount of time than the worn inserts. TT values decreases as wear level increases.

- Ratio: Ratio is the indicator of transition between non chaotic to chaotic states. High ratio indicates presence of transition to chaotic state, and low ratio represents quasi steady state. It is almost constant for all the three cases and has low value around 2.2, thus indicating the overall milling operation during cutting condition I is in quasi steady state. This is due to cutting operation is carried out at lower speed $300 \mathrm{rpm}$.

- Entropy: Entropy is also decreasing as wear values decreases. Entropy is an indicator of the amount of information required to identify the system. For sharp insert, entropy is high and hence more information is required where as for other worn inserts less information is required as their entropy values are low.

- Maxline: Maxline also indicates periodicity of the process. High values of Maxline indicate process is periodic and low values indicate the process is chaotic. Overall Maxline value is high showing periodicity in this cutting condition. For sharp insert the value is high, and for other two worn inserts. Maxline values are low. Thus milling with sharp insert is more periodic than with worn inserts. As insert worns out periodicity in the signals decreases.

- Trend: Trend indicates the drift or stationarity of the signal. A high value of trend indicates drift in the signal and low value of trend indicates stationarity. For sharp insert, trend value is high indicating drift in the signal. The worn inserts show lower trend values indicating stationarity in the process comparatively. 


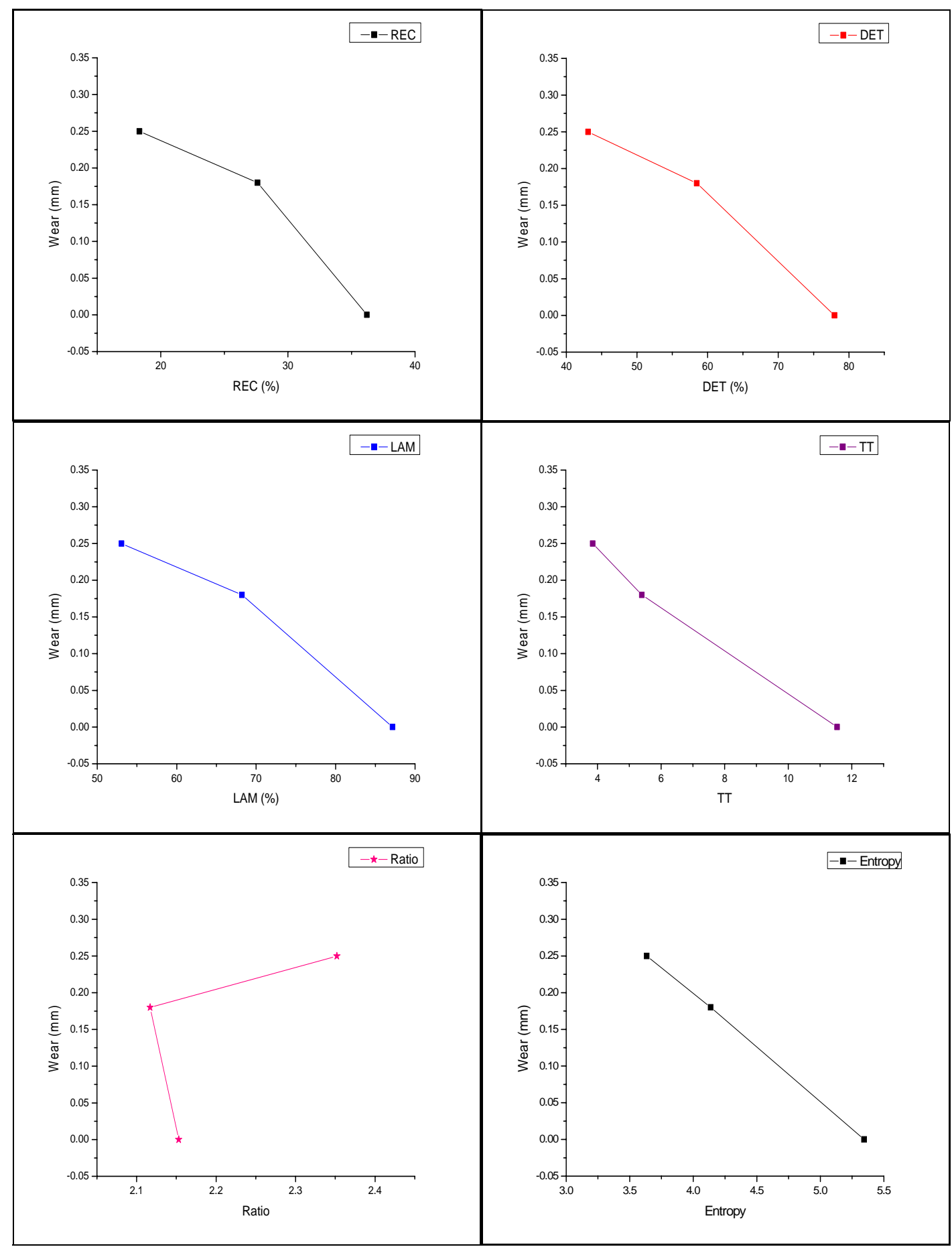

Figure 9: Influence of wear on RQA parameters during condition I

\subsubsection{Influence of wear on RQA parameter during cutting condition II}

Table 4 and Figure 10 shows the influence of wear on RQA parameters during cutting condition II. 
Table 4: RQA analysis for cutting condition II

\begin{tabular}{|c|c|c|c|}
\hline \multicolumn{5}{|c|}{ Cutting condition II } \\
\hline Tool State & sharp & $0.18 \mathrm{~mm}$ & $0.25 \mathrm{~mm}$ \\
\hline Percent recurrence & 0.744 & 0.465 & 0.189 \\
\hline Percent determinism & 7.919 & 1.63 & 1.786 \\
\hline Percent laminarity & 9.163 & 0.362 & 0 \\
\hline Trapping time & 3.375 & 2 & -1 \\
\hline Ratio & 10.644 & 3.51 & 9.473 \\
\hline Entropy & 2.286 & 1.585 & 2 \\
\hline Maxline & 12 & 4 & 0.347 \\
\hline Trend & -1.928 & 0.405 & \\
\hline
\end{tabular}

- REC: The recurrence values are very low for cutting condition II compared to cutting condition I, indicating low recurrence of the embedded state. This shows periodicity has drastically reduced as milling cutting conditions are changed with the increase in speed.

- DET: DET values are very low indicating less deterministic nature. There is no specific trend in DET values with respect to wear levels as seen in DET plot shown in Figure 10. The deterministic nature is reduced due to increase in cutting speed from $300 \mathrm{rpm}$ to $500 \mathrm{rpm}$. Deterministic nature during milling with sharp insert is better than for worn insert. This could be attributed to milling at higher speed.

- LAM: LAM values indicate intermittency or laminarity in the process which is the alternation of phases of apparently periodic and chaotic dynamics. LAM values for the cutting condition II show decreasing trend as wear increases. Since REC values are very low and LAM values are also very low it indicates dynamics process in this cutting condition is more chaotic and less periodic compared to cutting condition I. This is due to increase in cutting speed to 510 rpm where milling process is more complex.

- TT: TT values show a decreasing trend as wear values increase. In this case trapping time is also low indicating system is trapped for lower value of time compared to cutting condition I. It changes to -1 for $0.25 \mathrm{~mm}$ of flank wear. This is due to the increase of speed from $300 \mathrm{rpm}$ to $510 \mathrm{rpm}$ where milling process is becoming complex and system dynamics are changing fast due to its faster intermittent cutting.

- Ratio: The Ratio values are high compared to cutting condition I which indicates high chaotic machine dynamics during cutting condition II. The Ratio values as seen from the plot in Figure 10 do not show significant trend. For sharp insert and for $0.25 \mathrm{~mm}$ worn insert it is higher then $0.18 \mathrm{~mm}$ worn insert. For $0.18 \mathrm{~mm}$ worn insert it had higher DET value hence less chaotic.

- Entropy: Entropy also decreases as wear values decrease. Entropy is an indicator of the amount of information required to identify the system. It is decreasing with increase in wear level indicating decrease in information requirement as wear increases.

- Maxline: The values of Maxline are lower compared to that of cutting condition I. In general cutting process in this case is chaotic. Maxline parameter decreases as wear value decreases. For $0.25 \mathrm{~mm}$ worn insert it is highly chaotic compared to sharp insert. With increased speed and high wear value the milling dynamics tends to be complex and chaotic.

- Trend: Trend values for all the cases are very low which indicates there is no drift in the signal. This is basically due to change in milling cutting condition which has affected its dynamics to a great extent. 


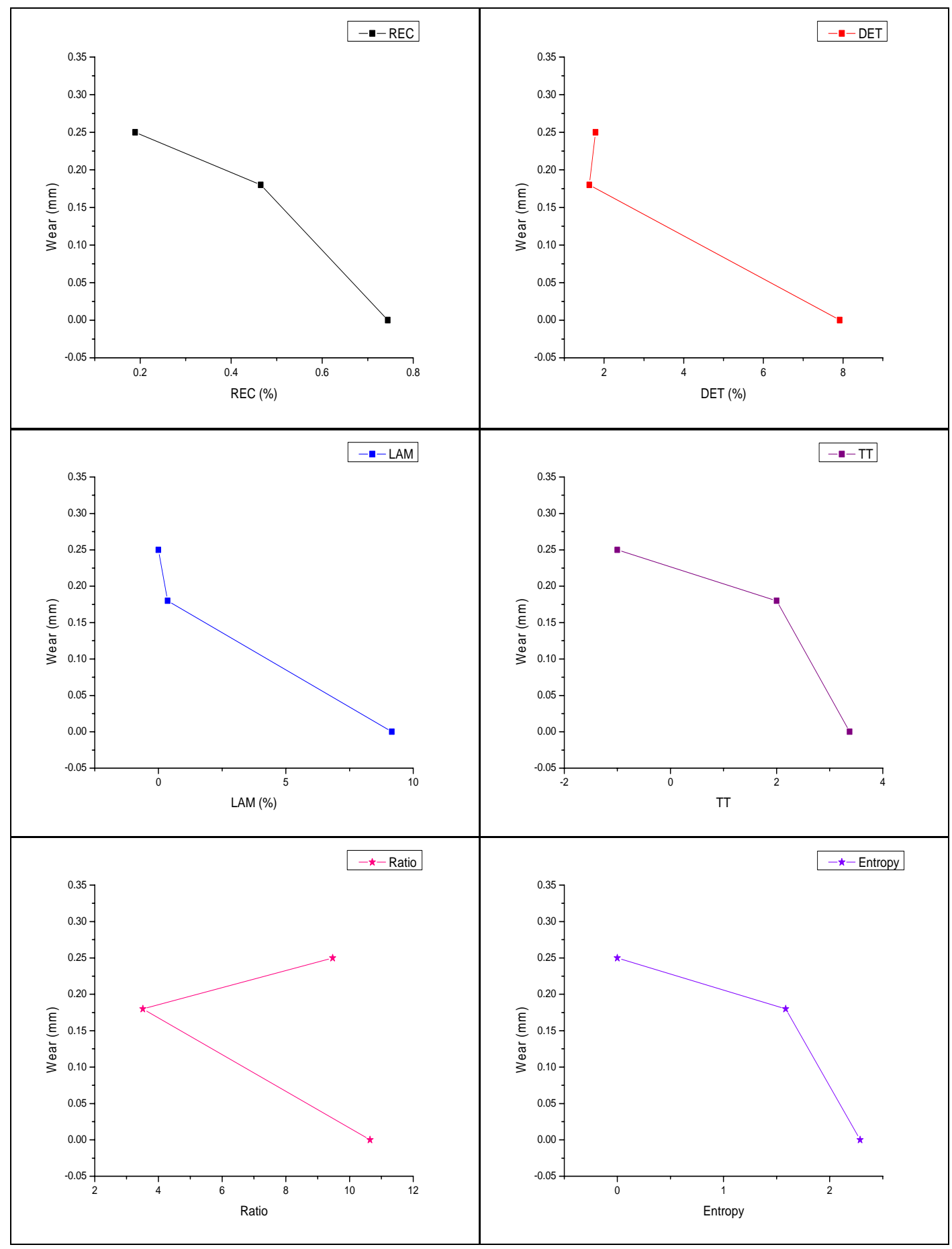

Figure 10: Influence of wear on RQA parameters during condition II

The RQA parameter plots have given insight to the underlying dynamics during face milling with different worn inserts. Most of the RQA parameters show a decreasing trend as wear values are increasing. This shows that RQA parameters are good indicators in detecting flank wear in face milling operation.

\section{Conclusions}

The feasibility of RQA to detect flank wear in face milling has been investigated. Both qualitative and quantitative approaches were used in order to detect tool wear. In the qualitative approach, the RP, could make distinction between sharp and wear inserts 
based on the color patterns. RP was parameterized to give RQA parameters. Most of these parameters were able to show definite trend in RQA parameters with variation in insert wear. In this investigative study the following conclusions were drawn:

- RP and RQA had significant features to distinguish wear states of the inserts.

- These techniques have special feature of analyzing non linear time series of vibration signal without actually making assumption of linearity in the time series and also not assuming on the noise factor.

- The RP plots based on color pattern and texture were able to distinguish wear levels through visualization in the qualitative investigation.

- In quantitative investigation for condition I the REC, DET, LAM, TT and Entropy showed decreasing trend as wear level increased. For condition II REC, TT, LAM and Entropy decreased as insert wear increased. In general REC, TT, LAM and Entropy for both conditions showed definite trend and hence can be used in detecting insert wear in face milling.

This investigative study deduced the potentiality of RP and RQA in detecting flank wear in face milling.

RQA technique has determinism factor which in time series data is not readily available to many approaches especially methods involving linear transformation of the data (e.g. the Fourier transform). RQA parameters posses greater sensitivity to changing dynamics than do linear approaches such as frequencies (Nichols et al, 2006).

It should be noted that RP and RQA techniques are methods which uses a small number of sampling points consisting of 100500 points depending on the characteristic dynamic response and the sampling rate (Marwan et al, 2007) (Litak et al, 2010). We think that this method can easily be adopted for monitoring of nonlinear cutting processes. Its seems this method have advantage as its explores all necessary information inside the given interval using this method one may consider feedback mechanism which will help in replacement of worn insert when critical wear sets in. In summary we can say that we propose a new method of detection of flank wear during milling process by the recurrence plots and quantification technique applied to time series of vibration signal.

\begin{tabular}{ll}
\multicolumn{2}{l}{ Nomenclature } \\
RP & Recurrence Plot \\
RQA & Recurrence Quantification Analysis \\
AMI & Average Mutual Information \\
FNN & False Nearest Neighbor \\
$\tau$ & Delay time \\
$m$ & Embedded dimension \\
REC & Percent recurrence \\
DET & Percent determinism \\
LAM & Percent laminarity \\
TT & Trapping Time \\
ENT & Entropy
\end{tabular}

\section{Acknowledgement}

The authors would like to thank the Technical Education Quality Improvement Programme (TEQIP) of the Government of India for the funding of the NI Data Acquisition system and LabVIEW software under the scheme of "Modernization of laboratories and workshops" to carry out the research work.

\section{References}

Belaire-Franch J., 2004. Testing for non-linearity in an artificial financial market: a recurrence quantification approach, Journal of Economic Behavior \& Organization. Vol 54, No.4, pp. 483-494.

Dimla D.E. Sr. 2000. Sensor signals for tool wear monitoring in metal cutting operations-review of methods. International Journal of Machine Tools \& Manufacture, Vol. 40, pp. 1073-1098.

Dissinger B.M., Rohde G.K., Rhodes R.B., Bucholtz Jr.F. 2006. Plots for the detection of deterministic signals in noise. Document report from Naval Research Laboratory, 4555 Overlook Avenue, SW Washington, DC.

Eckmann J.P., Kamphorst S.O., Ruelle D., 1987. Recurrence plots of dynamical systems. Europhys. Lett.,Vol 5, pp. 973-977.

Elwakil A.S., Soliman A.M., 1999. Mathematical models of the twin-T, wien-bridge and family of minimum component electronic chaos generators with demonstrative recurrence plots. Chaos Solit. Fract. Vol 10, No.8, pp.1399-1411.

Eugene Kononov's VRA software available at http://home.netcom.com/eugenek/download.htm

Fraser A., Swinney H., 1986. Independent coordinates for strange attractors from mutual information. Phys Rev A. Vol 33, pp. 1134-1140. 
Gonzáleza N.A., Ochoa E.G. and Sánchez J.G., 2008. Assessment of the dynamics of corrosion fatigue crack initiation applying recurrence plots to the analysis of electrochemical noise data. Inter. Journal of Fatigue, Vol 30, No.7, pp.1211- 1219.

Karuda S., Bradley C., 1997. A review of machine vision sensors for tool condition monitoring. Computers in Industry. Vol 34, pp $55-72$.

Kennel M., Brown R., Abarbanel H., 1992. Determining embedding dimension for phase space reconstruction using a geometrical construction”. Phys Rev A. Vol 45 pp. 3403-3411.

Litak G., Gajewski J., Syta A., Jonak J., 2008. Quantitative estimation of the tool wear effects in a ripping head by recurrence plots. Journal of Theoretical and Applied Mechanics. Vol 46, No.3, pp. 521-530.

Litak G., Wiercigroch M., Horton B.W., Xu X., 2010. Transient chaotic behaviour versus periodic motion of a pendulum by reccurance plots. Z Angew Math Mech Vol 90, No. 1, pp. 33 - 41.

Marwan N., 2003. Encounters with neighbours: current development of concepts based on recurrence plots and their applications. $\mathrm{PhD}$ Thesis, University"at Potsdam, Potsdam

Marwan N., Romano M.C.,Thiel M., Kurths J., 2007. Recurrence plots for the analysis of complex system. Phys Report Vol 438, pp. 237-329.

Masao M. 2006. Recurrence plot-based approach to the analysis of IP-network traffic in terms of assessing non-stationary transitions over time. IEEE Transactions on Circuits and Systems. Vol. 53, No. 10, pp. 2318 - 2326.

Matassini L., Manfredi C. 2002. Software corrections of vocal disorders. Comput. Meth. Programs Biomed. Vol 68, No.2, pp. 135-145.

Nichols J.M., Trickey S.T. and Seaver M. 2006 Damage detection using multivariate recurrence quantification analysis. Mech. Systems and Signal Processing. Vol 20, pp. 421-437.

Sen A.K., Longwic R., Litak G.and Górski K. 2008. Analysis of cycle-to-cycle pressure oscillations in a diesel engine. Mech. Systems and Signal Processing. Vol 22, pp. 362-373.

Sick B. 2002. On-line and indirect tool wear monitoring in turning with artificial neural networks: a review of more than a decade of research. Mechanical Systems and Signal Processing. Vol 16, No.4, pp. 487-546.

Webber C.L. Jr., Zbilut J.P. 1994. Dynamical assessment of physiological system and status using recurrence plot strategies. Journal of Applied Physiology. Vol 76, pp. 965-973.

Zbilut J.P. and C.L. Webber, Jr., 1992. Embeddings and delays as derived from quantification of recurrence plots. Physics Letters A. Vol 171, pp. 199-203.

\section{Biographical notes}

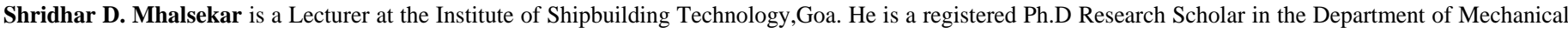

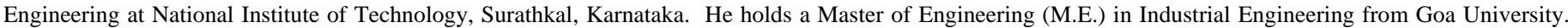
His research interests are in Manufacturing engineering.

Dr. Shrikantha S. Rao is an Associate Professor in the Department of Mechanical Engineering at National Institute of Technology, Surathkal, Karnataka. He holds a Ph.D. degree in Application of NueroFuzzy Systems in Intelligent Manufacturing. His research interests are in the areas of Process modeling using artificial intelligence techniques like Expert Systems, Artificial Neural Networks, Fuzzy logic and Genetic Algorithm. He has more than thirty research publications to his credit.

Dr. K. V. Gangadharan is an Associate Professor in the Department of Mechanical Engineering at National Institute of Technology, Surathkal, Karnataka. He holds a Ph.D. degree in Railroad vehicle dynamics from IIT Madras. His research interest are in the areas of vibration and its control, smart material and its application in vibration control, dynamics, finite element analysis, condition monitoring and experimental methods in vibration. He is actively engaged in industrial consultancy and sponsored research projects and has more than thirty research publications to his credit.

Received May 2010

Accepted May 2010

Final acceptance in revised form June 2010 